○

\title{
Case Series on Initial Responses to Intravitreal Brolucizumab in Patients with Recalcitrant Chronic Wet Age-Related Macular Degeneration
}

This article was published in the following Dove Press journal: International Medical Case Reports Journal

Jaycob Avaylon (1)'

Sol Lee ${ }^{2}$

Ron P Gallemore ${ }^{2}$

'California Northstate University, College of Medicine, Elk Grove, CA, USA; ${ }^{2}$ Retina Macula Institute, Torrance, CA, USA
Correspondence: Ron P Gallemore Retina Macula Institute, 420I Torrance Blvd., Ste 220, Torrance, CA 90503, USA Tel + I 310 4l3-7020

Email rongallemoremd@gmail.com
Purpose: To report a case series of initial responses to intravitreal brolucizumab in patients already undergoing anti-VEGF therapy for wet age-related macular degeneration.

Case Series: Six eyes (6 patients) with a history of wet age-related macular degeneration presented with either decline in vision or no improvement while undergoing treatment with anti-VEGF therapy - aflibercept or bevacizumab. Patients were switched to intravitreal brolucizumab. Four weeks post-injection, there was no significant change in visual acuity. Optical coherence tomography scans were taken and improved IRF/SRF, central macular thickness and average pericentral thickness were observed in all 6 patients. No serious adverse reactions were observed, including signs of vasculitis or increase in anterior chamber cell count at the 4-week follow-up for all 6 patients.

Conclusion: Intravitreal brolucizumab appears to be a safe and limitedly effective option for patients with recalcitrant CNV from wet AMD.

Keywords: macular degeneration, anti-VEGF, brolucizumab, wet AMD, choroidal neovascularization

\section{Introduction}

Anti-vascular endothelial growth factor (anti-VEGF) therapy has now become the standard of care for the treatment of neovascular (wet) age-related macular degeneration (wet AMD), as well as other leading causes of vision loss, including diabetic retinopathy, retinal vein occlusion, and cystoid macular edema. ${ }^{1}$ Over the past decade, different anti-VEGF agents have been developed to improve treatment outcomes. In 2004, the FDA approved the first drug for wet AMD: pegaptanib (Macugen, OSI Pharmaceuticals, New York, NY). However, its use declined after the development of two more effective agents, ranibizumab (Lucentis, Novartis, East Hanover, NJ), which was FDA approved for wet AMD in 2006, and the cheaper off-label drug, bevacizumab (Avastin, Genentech, South San Francisco, CA). ${ }^{1}$

Ranibizumab was administered on a monthly regimen for 1 year and up to 2 years in clinical trials. ${ }^{2}$ Aflibercept (Eylea, Regeneron, Tarrytown, NY), was subsequently developed with the hope of extending the treatment interval and was FDA-approved in 2011 for treatment of wet AMD with a treatment regimen of every 4 weeks for the first 12 weeks, then once every 8 weeks thereafter. ${ }^{3}$ The increased efficacy was found to be the result of broader blockade of VEGF receptors (VEGFR-1 and VEGFR-2). ${ }^{4}$ Ranibizumab is a $48-\mathrm{kDa}$ recombinant humanized IgG1 kappa isotype monoclonal 
antibody against VEGF-A, most effective against isoforms VEGF121, VEGF165, and VEGF189. ${ }^{4}$ Aflibercept (115 $\mathrm{kDa}$ ) is a fusion protein composed of the binding domains of human VEGF receptors (VEGFR-1 and VEGFR-2) fused with the Fc region of IgG1, acting as a decoy receptor and has a broader coverage including VEGF-A, VEGF-B and placental growth factor (PGF). ${ }^{4}$

Recently, brolucizumab (Beovu, Novartis, East Hanover, NJ) was developed with the hope of further extending treatment intervals. Currently, brolucizumab is FDA-approved for 3 monthly injections followed by a 2-to 3-month maintenance dose. ${ }^{5-7}$ HAWK and HARRIER Phase III clinical trials showed non-inferiority and comparable efficacy to aflibercept, but with the advantage of a longer maintenance dose interval of every 12 weeks. ${ }^{6,7}$ Brolucizumab is a $26 \mathrm{kDa}$ humanized single-chain antibody fragment that inhibits all isoforms of VEGF-A, including VEGF110, VEGF121, and VEGF165, by preventing interactions with VEGFR-1 and VEGFR-2. ${ }^{6,7}$

Brolucizumab was made available in October 2019. We report initial results of this medication for the treatment of persistent active choroidal neovascularization (CNV) associated with AMD in patients with either a decline or no improvement in vision while undergoing treatment with previously FDA-approved anti-VEGF agents.

\section{Methods}

Retrospective chart review of six eyes (six patients) treated for wet macular degeneration. Best-corrected Snellen visual acuity (BCVA), central macular thickness (CMT), and average pericentral thickness (APT) using Cirrus OCT Model 5000 (Zeiss Medical Technology, United States) were measured, as well as any adverse effects.

\section{Case Series}

\section{Case I}

An 87-year-old Caucasian female with a history of persistent choroidal neovascularization associated with agerelated macular degeneration was treated with monthly anti-VEGF intravitreal injections. The right eye reached the end-stage vision of $\mathrm{CF}$ at $3 \mathrm{ft}$, and the left eye BCVA has been fluctuating from $20 / 25$ to $20 / 40$ over the last 5 years. The patient was on monthly aflibercept (Eylea, Regeneron, Tarrytown, NY) intravitreal injections in both eyes; however, the right eye was switched to bevacizumab (Avastin, Genentech, South San Francisco, CA) with hopes of discontinuing treatment. The left eye was maintained on aflibercept until it developed increased macular edema and was then switched to bevacizumab with the reasoning that the eye was developing tachyphylaxis to aflibercept. Macular edema improved on bevacizumab and was maintained for 5 monthly injections until the macular edema began to worsen. The left eye was switched back to aflibercept and has been maintained on a monthly interval for the past 1.5 years (Figure 1A). The patient agreed to start monthly brolucizumab (Beovu, Novartis, East Hanover, NJ) intravitreal injections in her left eye in hopes of extending her current treatment

A

A

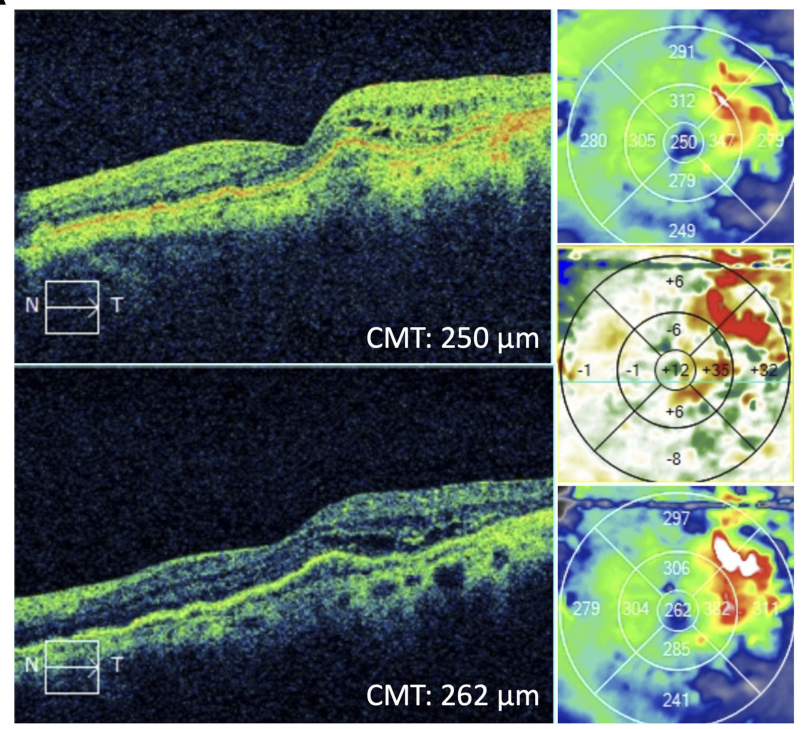

B

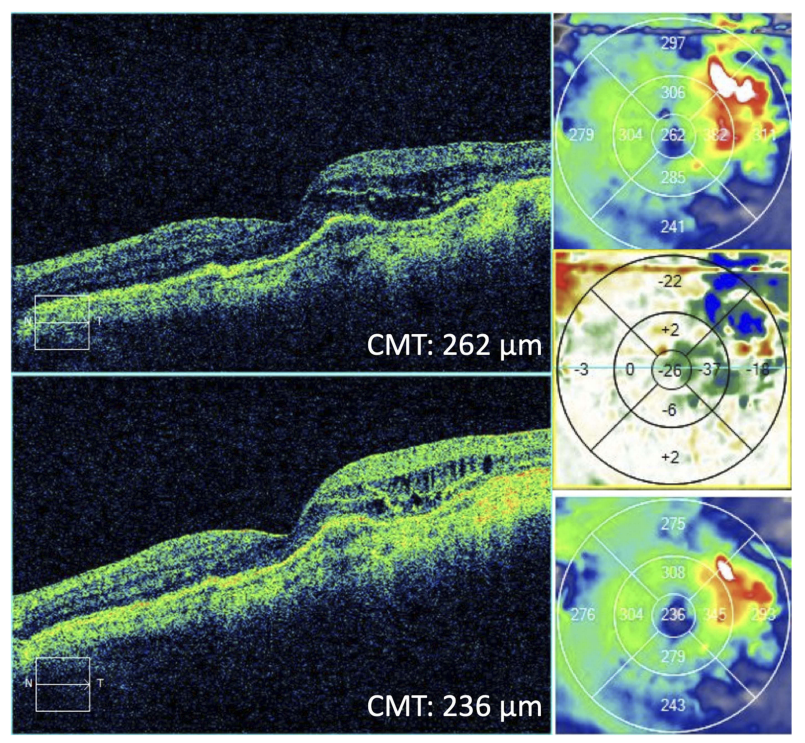

Figure I Case I. (A) Before and after OCT images of the patient after 3 intravitreal aflibercept injections every 4 weeks. (B) Before and after OCT image I month after I intravitreal brolucizumab injection. 
interval of every 4 weeks to every $8-12$ weeks seen with brolucizumab. Baseline BCVA was 20/30 OS. At 4 weeks post-injection, her vision was 20/40 OS. Optical coherence tomography (OCT) studies showed improved IRF, and the CMT decreased by 26 microns from the pre-brolucizumab baseline (Figure 1B, Table 1).

\section{Case 2}

A 78-year-old Caucasian female with a history of dry macular degeneration presented with sudden vision distortion in the right eye and a BCVA of 20/200. Fluorescein angiogram showed occult CNV with late leakage and OCT studies showed increased central foveal thickness along with subretinal fluid. The patient was started on monthly aflibercept intravitreal injections. BCVA improved and stabilized to 20/40 for 5 months. Treatment intervals were increased to 5 weeks, but BCVA dropped to $20 / 80$, and the interval was reduced back to 4 weeks. However, at 4 weeks, vision did not improve. Repeat fluorescein and indocyanine green angiograms showed persistent occult CNV and leakage on OCT (Figure 2A). The patient agreed to start monthly brolucizumab intravitreal injections.

Table I Summary of Visual Acuity and OCT Macular Thickness Changes in Patients Undergoing Treatment of Recalcitrant Chronic Wet AMD with Intravitreal Brolucizumab

\begin{tabular}{|c|c|c|c|c|c|}
\hline $\begin{array}{l}\text { Case } \\
\text { No. }\end{array}$ & Timeline & VA/PH & $\begin{array}{l}\text { Change in VA } \\
\text { Baseline - I } \\
\text { Month }\end{array}$ & CMT & APT \\
\hline 1 & $\begin{array}{l}\text { Baseline } \\
1 \text { month s/p }\end{array}$ & $\begin{array}{l}20 / 30+1 \\
20 / 40\end{array}$ & $-0.13 \log M A R$ & $\begin{array}{l}262 \\
236\end{array}$ & $\begin{array}{l}319 \\
309\end{array}$ \\
\hline 2 & $\begin{array}{l}\text { Baseline } \\
2 \text { weeks s/p } \\
\text { I month s/p }\end{array}$ & $\begin{array}{l}20 / 80 \\
20 / 40+I \\
20 / 50-I / \mathrm{NI}\end{array}$ & $+0.20 \log M A R$ & $\begin{array}{l}234 \\
219 \\
220\end{array}$ & $\begin{array}{l}244 \\
236 \\
240\end{array}$ \\
\hline 3 & $\begin{array}{l}\text { Baseline } \\
2 \text { weeks s/p } \\
1 \text { month s/p }\end{array}$ & $\begin{array}{l}20 / 400 / 200 \\
20 / 400 / \mathrm{NI} \\
20 / 200\end{array}$ & $0.00 \log M A R$ & $\begin{array}{l}237^{*} \\
268^{*} \\
285^{*}\end{array}$ & $\begin{array}{l}356 \\
339 \\
341\end{array}$ \\
\hline 4 & $\begin{array}{l}\text { Baseline } \\
2 \text { weeks s/p } \\
1 \text { month s/p }\end{array}$ & $\begin{array}{l}20 / 40 \\
20 / 60 / 50 \\
20 / 60-1\end{array}$ & $-0.18 \log M A R$ & $\begin{array}{l}293 \\
241 \\
236\end{array}$ & $\begin{array}{l}313 \\
292 \\
290\end{array}$ \\
\hline 5 & $\begin{array}{l}\text { Baseline } \\
2 \text { weeks s/p } \\
1 \text { month s/p }\end{array}$ & $\begin{array}{l}20 / 200 \\
20 / 200 \\
20 / 200 / \mathrm{NI}\end{array}$ & $0.00 \log M A R$ & $\begin{array}{l}507 \\
498 \\
489\end{array}$ & $\begin{array}{l}482 \\
471 \\
424\end{array}$ \\
\hline 6 & $\begin{array}{l}\text { Baseline } \\
2 \text { weeks s/p } \\
1 \text { month s/p }\end{array}$ & $\begin{array}{l}20 / 100-2 \\
20 / 200 \\
20 / 200\end{array}$ & $-0.3 \log M A R$ & $\begin{array}{l}200 \\
181 \\
183\end{array}$ & $\begin{array}{l}267 \\
252 \\
257\end{array}$ \\
\hline
\end{tabular}

Notes: *Artifact present over fovea on OCT scans, APT was used instead for monitoring.

Abbreviations: CMT, central macular thickness (um); APT, average pericentral thickness: average thickness (um) of the 4 quadrants adjacent to the central measured region.
BCVA was 20/80. At 2 weeks post-injection, BCVA improved to $20 / 40$ and her CMT improved by 15 microns. At 4 weeks post-injection, her vision was 20/50 OD. OCT showed improved SRF, and the CMT decreased by 14 microns from the pre-brolucizumab baseline (Figure 2B, Table 1).

\section{Case 3}

A 62-year-old Caucasian male was referred for a macular hole in his phakic left eye. Initial BCVA was CF at $6 \mathrm{ft} \mathrm{OS.} \mathrm{On}$ exam, a full-thickness stage III macular hole was noted along with a stellate pattern pigment nasal to the fovea associated with an irregular PED confirmed on OCT. Lacy hyperfluorescence with late leakage was found on fluorescein angiogram, and possible hotspot and polyp were found on indocyanine green angiogram (not shown). This is consistent with classic $\mathrm{CNV}$, a rare association with a macular hole. ${ }^{8}$ The patient was treated with bevacizumab intravitreal injection, and macular edema slightly improved on OCT. The patient then underwent vitrectomy, membrane stripping, and placement of $\mathrm{C} 3 \mathrm{~F} 8$ gas tamponade. This was delayed by 1 month after initial consultation as the patient was not able to be cleared for surgery. The hole appeared closed on clinical exam 1 day post-operatively. Four weeks post-operatively, the macular hole reopened, and the patient underwent repeat surgery with placement of silicone oil tamponade the following week. After the second surgery, the hole was closed but once again reopened after 4 weeks. Fluorescein and indocyanine green angiograms showed occult CNV with active leakage. The patient was started on monthly aflibercept intravitreal injections and completed 3 treatments before being lost to follow-up for 5 months due to ankle surgery. On return, BCVA was unchanged at $\mathrm{CF}$ OS, and OCT showed an increase in macular edema. The patient was restarted on monthly aflibercept injections. However, after 5 treatments with aflibercept, OCT showed increased edema, and the patient was started on brolucizumab (Figure 3A). Baseline BCVA was 20/200 OS. At 2 weeks postinjection, BCVA was 20/400 and average pericentral macular thickness (APT) decreased by 17 microns. At 4 weeks postinjection, BCVA returned to 20/200 OS. OCT showed improved IRF, and the APT decreased by 15 microns from the pre-brolucizumab baseline (Figure 3B, Table 1).

\section{Case 4}

A 58-year-old Caucasian male was lost to follow-up for 3 years and returned complaining of decreasing vision in his right eye. BCVA before being lost to follow-up was 20/502 OD. He was previously being treated for wet macular degeneration with anti-VEGF intravitreal injections. 
A

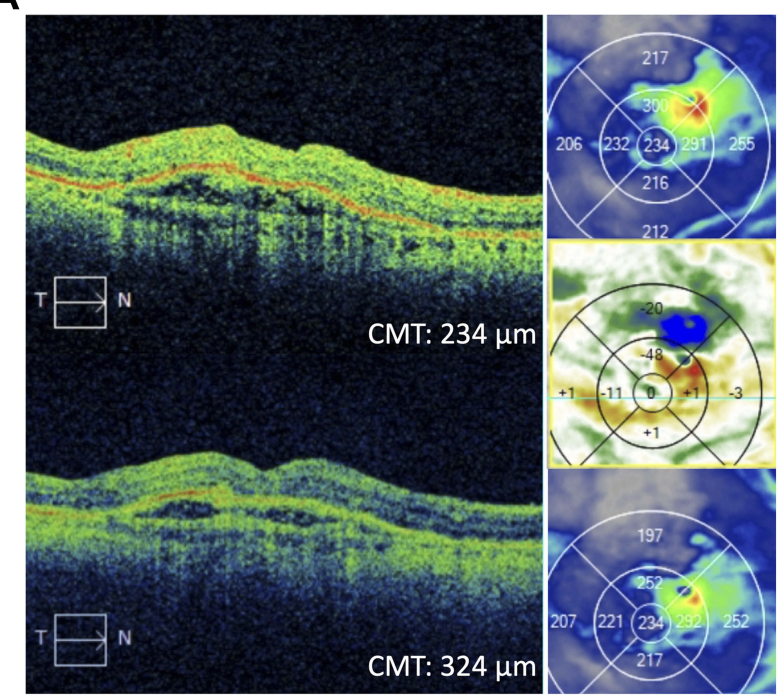

B

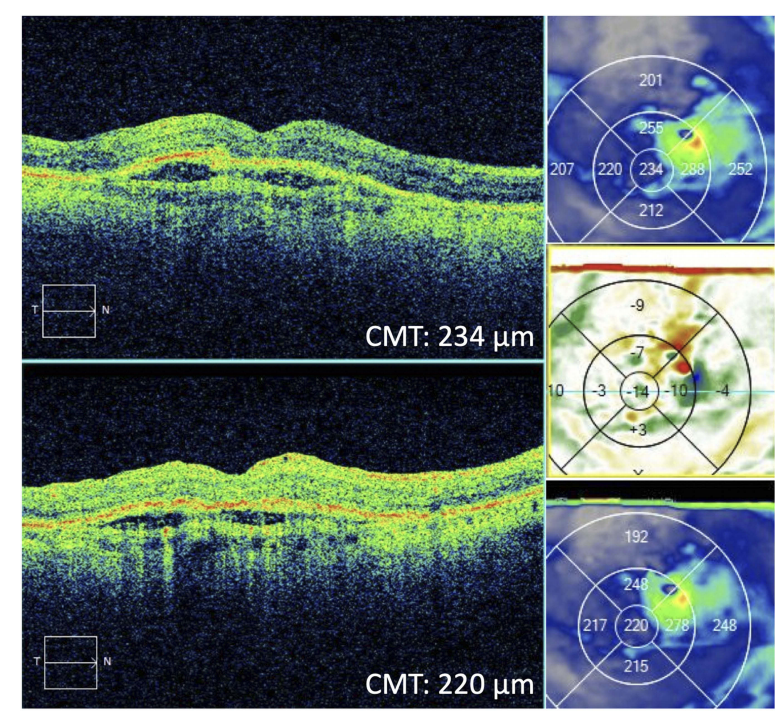

Figure 2 Case 2. (A) Before and after OCT images of the patient after 4 intravitreal aflibercept injections every 4 weeks. (B) Before and after OCT image I month after I intravitreal brolucizumab injection.

BCVA on return dropped to 20/100 OD. Fluorescein angiogram showed $\mathrm{CNV}$ with $\mathrm{CME}$ and the patient was restarted on monthly bevacizumab intravitreal injections. The patient, however, returned for four injections at 2- to 3-month intervals since he felt his vision was stable. During this time, BCVA fluctuated between 20/100 and 20/60 OD. The patient was then lost to follow-up for 21 months. On return, his BCVA was 20/40 OD; however, fluorescein/indocyanine green angiograms and OCT showed progressive fibrovascular changes along with IRF (Figure 4A). The patient was started on brolucizumab intravitreal injections. At 2 weeks post-injection, his BCVA was 20/50 OD, and CMT improved by 52 microns
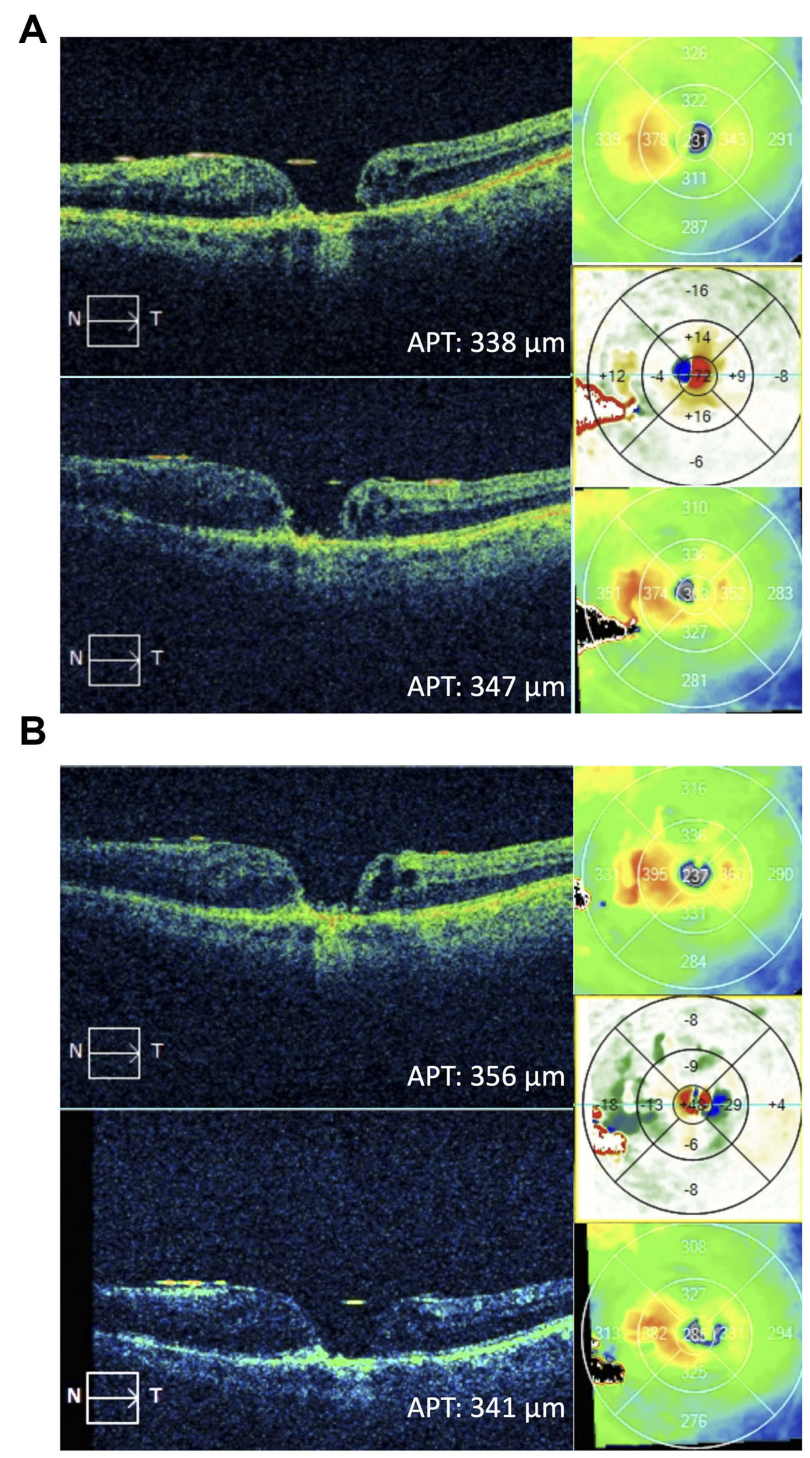

Figure 3 Case 3. (A) Before and after OCT images of the patient after 3 intravitreal aflibercept injections every 4 weeks. (B) Before and after OCT image I month after I intravitreal brolucizumab injection.

(Table 1). At 4 weeks post-injection, VA was 20/60 OD. OCT showed significantly improved IRF, and the CMT decreased by 57 microns compared to pre-brolucizumab baseline (Figure 4B, Table 1).

\section{Case 5}

A 68-year-old Asian male with a complicated history of high myopia, recurrent CME-induced macular hole x 2 OS, and wet AMD OS was treated with monthly aflibercept intravitreal injections for the past 2 years. The patient also developed chronic uveitis OS and received 3 Ozurdex implants over the course of 3 years. The patient's VA remained stable at 20/200 OS during this time period. Recently, the patient was treated with one fluocinolone 

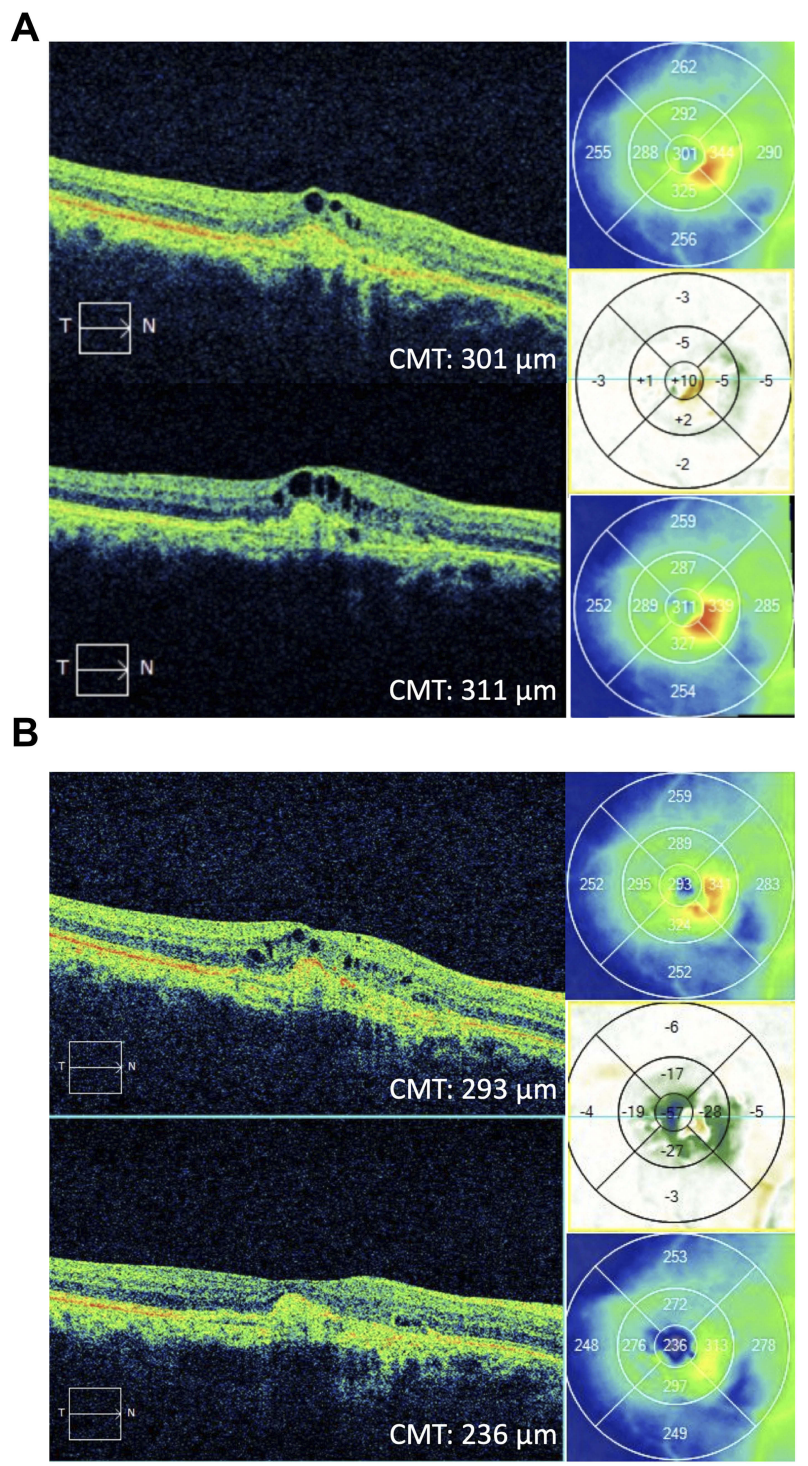

Figure 4 Case 4. (A) Before and after OCT images of the patient after 3 intravitreal bevacizumab injections every 2-3 months. (B) Before and after OCT images I month after I intravitreal brolucizumab injection.

acetonide intravitreal implant (Yutiq, Eyepoint Pharmaceuticals, Watertown, MA) injection for chronic uveitis, in addition to his regular monthly aflibercept intravitreal injections for wet AMD. Three months later, VA remained unchanged at 20/200 OS. OCT showed no improvement in macular edema over the last 3 months and irregular thickening of the choroid (Figure 5A), as well as some focal hyperfluorescence with late cystoid macular edema on fluorescein angiogram (not shown). CNV was diagnosed but an element of inflammatory edema could not be ruled out. In hopes of extending his treatment interval and possibly improving vision, the patient agreed to start brolucizumab intravitreal injections. At 2 weeks post-injection, vision remained stable at 20/

\section{A}

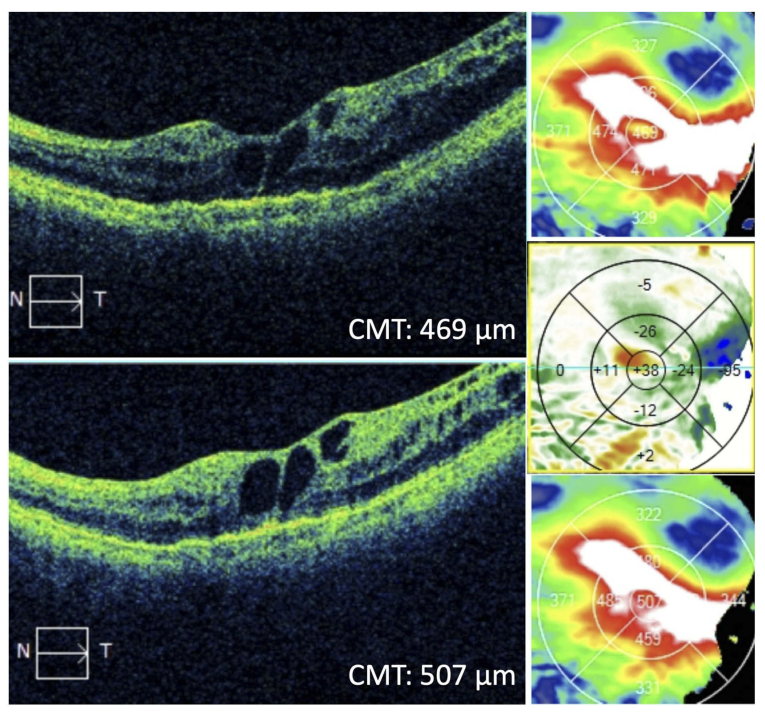

B

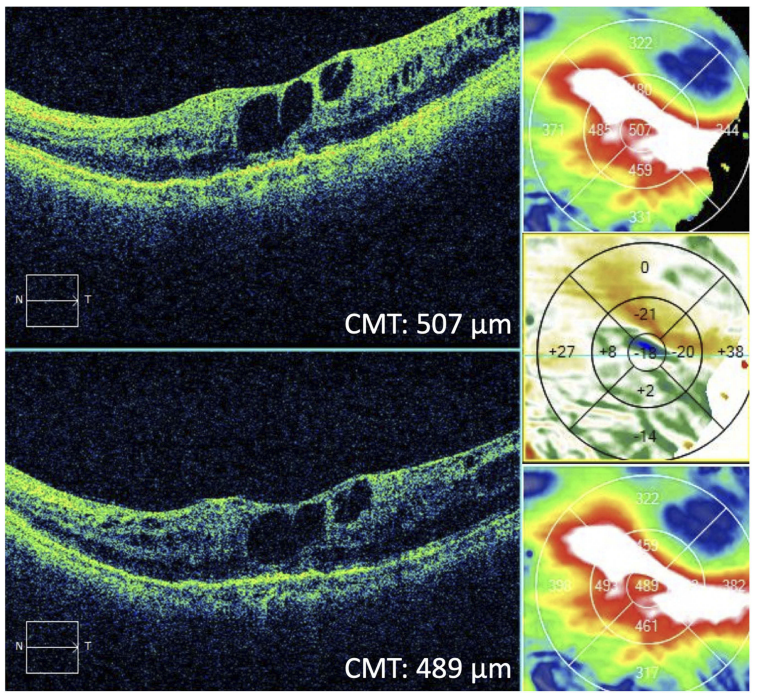

Figure 5 Case 5. (A) Before and after OCT images of the patient after 4 intravitreal aflibercept injections every 4 weeks. (B) Before and after OCT images I month after I intravitreal brolucizumab injection.

200 OS and CMT improved by 9 microns (Table 1). At 4 weeks post-injection, VA remained stable at 20/200 OS. OCT showed slightly improved IRF, and the CMT decreased by 18 microns compared to pre-brolucizumab baseline (Figure 5B, Table 1).

\section{Case 6}

An 89-year-old patient with a 10-year history of wet macular degeneration in both eyes was initially treated with monthly bevacizumab intravitreal injections $\mathrm{OU}$ and then switched to monthly aflibercept intravitreal injections OU. VA fluctuated between 20/50 and 20/80 OU 
until the patient had a flare-up of new leakage on fluorescein angiogram in both eyes and was treated with combination therapy of monthly aflibercept intravitreal injection and yearly verteporfin photodynamic therapy in both eyes. The patient has also been on difluprednate $0.5 \%$ ophthalmic drops (Durezol, Novartis, East Hanover, NJ), bromfenac $0.075 \%$ ophthalmic drops (Bromsite, Sun Pharmaceutical Industries, Princeton, NJ) as an adjunct therapy for the recalcitrant edema. The patient's VA has been stable at 20/80 OS on monthly aflibercept intravitreal injections for 4 treatments OU until recently when the patient's VA OS dropped to 20/100. OCT showed increased macular thickness, appreciated on OCT difference map (Figure 6A). The patient was then started on

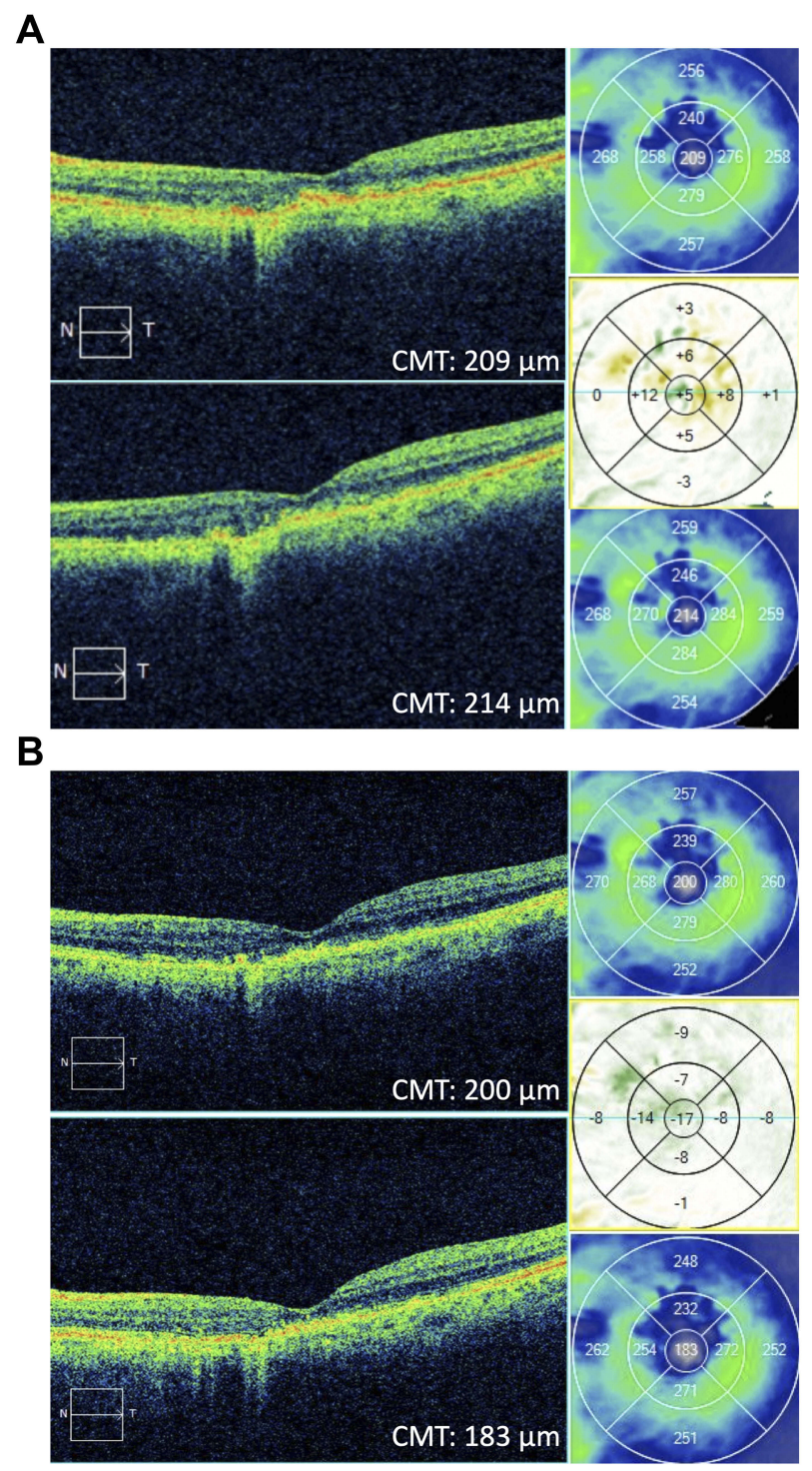

Figure 6 Case 6. (A) Before and after OCT images of the patient after I intravitreal aflibercept injection. (B) Before and after OCT images I month after I intravitreal brolucizumab injection. brolucizumab intravitreal injections OS. At 2 weeks postinjection, the patient's vision decreased to 20/200 OS; however, OCT showed a CMT reduction of 19 microns (Table 1). At 4 weeks post-injection, VA remained stable at 20/200 OS. OCT showed slightly improved central IRF, and the CMT improved by 17 microns compared to pre-brolucizumab baseline (Figure 6B, Table 1).

\section{Discussion}

Brolucizumab (Beovu, Novartis, East Hanover, NJ) was FDA approved for the treatment of wet AMD on October 8, 2019. In our case series, we utilize the drug to treat patients with persistent activity while on other antiVEGF agents. We observe improved responses with decreased intraretinal fluid and/or subretinal fluid when present, as well as decreased CMT and APT in all cases. This improvement could well be due to the increased penetrance into retinal tissues owing to its smaller 26 $\mathrm{kDa}$ size compared to ranibizumab (48 $\mathrm{kDa}$ ), aflibercept (115 kDa), and bevacizumab (149 kDa). ${ }^{9,10}$ The small size, solubility, and stability also allow a greater dose to be injected $(6 \mathrm{mg})$ compared to ranibizumab $(0.5 \mathrm{mg}-$ indicated for wet AMD), aflibercept ( $2 \mathrm{mg})$, and bevacizumab (1.25mg). ${ }^{9}$ There is the argument, however, that tachyphylaxis may have been a factor and that another agent can, therefore, be more effective. Further studies will be required to rule this out.

Four weeks post-injection, measured visual acuity improved in 1 patient, remained stable in 2 patients, and decreased in 3 patients (Table 1). There may be a difference between the measured VA and a true change in VA due to factors such as patient motivation, fatigue, eye dryness, and variability in testing. ${ }^{11,12}$ The Snellen eye chart has been shown to have a large test-retest variability (TRV) of \pm 0.29 to $\pm 0.33 \log$ MAR. ${ }^{12}$ Since the maximum change in VA observed in our case series was -0.30 logMAR (Table 1), true improvement or worsening of visual acuity cannot be concluded, but can be considered as stable for all 6 patients.

All nonselective anti-VEGF drugs to date, bevacizumab, ranibizumab, and aflibercept, have been reported to cause an inflammatory response in a small subset of patients ranging from $1 \%$ to $4 \% .{ }^{13,14}$ Brolucizumab was found to have a higher rate of inflammation (4\%) compared to aflibercept (1\%) in the pivotal HAWK and HARRIER clinical trials. ${ }^{6} \mathrm{We}$, therefore, monitored each patient for an inflammatory response by counting the anterior chamber (AC) cells before, 2 weeks, and 4 
weeks after treatment. In this limited follow-up period, we observed no inflammatory cells at the 1 month follow-up for 5 of the 6 cases. The patient in case 5 had trace cells in the $\mathrm{AC}$; however, there is an underlying history of uveitis already controlled with treatment. We observed no increase in AC cell count for all 6 cases. Recently, there have been reports of retinal vasculitis associated with brolucizumab injections. To date, there have been 14 reported cases, 11 of which were classified as occlusive retinal vasculitis. ${ }^{15}$ We have not observed signs of vasculitis in the 6 cases.

A disadvantage of brolucizumab is the lack of prefilled syringes. Recently, both aflibercept and ranibizumab are supplied in a preloaded syringe, whereas brolucizumab is supplied in a single-dose vial which needs to be drawn into a syringe using a filter needle. Preloaded syringes offer a significant advantage over prior generations as they decrease the risk of contamination, such as during drawing of the medication. It is also delivered in silicone-free syringes, reducing the risk of silicone oil floaters. ${ }^{16}$

For now, we have found that brolucizumab has a limited positive response and is safe acutely for these patients with recalcitrant chronic wet AMD. Keep in mind that much of the edema was improved on prior chronic anti-VEGF therapy. While there were no significant changes in vision, further reduction in edema was still achieved as a rule in all cases with the addition of brolucizumab suggesting increased efficacy. This is, to our knowledge, the first case series utilizing this new agent in patients with recalcitrant chronic wet AMD. Longer-term studies will be reported to confirm the safety and efficacy of this new drug in such patients and whether or not we will be able to extend treatment intervals to 2-3 months. Historically, we have eliminated the extension interval in those patients that have been resistant to other drugs. A reduction of injection frequency will not only help improve the visual outcomes of patients who are unable to return for treatment on a consistent basis, but also certainly improve their quality of life.

\section{Patient Consent}

We confirm that any aspect of the work covered in this manuscript that has involved human patients has been conducted with the ethical approval of all relevant bodies. Informed written consent has been obtained from all patients to have case details and any accompanying images published. Institutional approval was not required to publish case details.

\section{Acknowledgments}

I have obtained all of my co-authors' full disclosures. There are no acknowledgements we would like to make. No institutional approval was required for the publishing of this case series.

\section{Author Contributions}

All authors attest that they meet the current ICMJE criteria for authorship. All authors made substantial contributions to conception and design, acquisition of data, or analysis and interpretation of data; took part in drafting the article or revising it critically for important intellectual content; gave final approval of the version to be published; and agree to be accountable for all aspects of the work.

\section{Funding}

No grant support or funding was given.

\section{Disclosure}

The authors report no conflicts of interest in this work.

\section{References}

1. Lanzetta P, Loewenstein A; Vision Academy Steering Committee. Fundamental principles of an anti-VEGF treatment regimen: optimal application of intravitreal anti-vascular endothelial growth factor therapy of macular diseases. Graefes Arch Clin Exp Ophthalmol. 2017;255(7):1259-1273. doi:10.1007/s00417-017-3647-4

2. Mitchell P, Korobelnik J, Lanzetta P, et al. Ranibizumab (Lucentis) in neovascular age-related macular degeneration: evidence from clinical trials. Br J Ophthalmol. 2010;94:2-13. doi:10.1136/bjo.2009.159160

3. Heier JS, Brown DM, Chong V, et al. Intravitreal aflibercept (VEGF Trap-Eye) in wet age-related macular degeneration. Ophthalmology. 2012;119(12):2537-2548. doi:10.1016/j.ophtha.2012.09.006

4. Papadopoulos N, Martin J, Ruan Q, et al. Binding and neutralization of vascular endothelial growth factor (VEGF) and related ligands by VEGF Trap, ranibizumab and bevacizumab. Angiogenesis. 2012;15 (2):171-185. doi:10.1007/s10456-011-9249-6

5. Beovu [Package Insert]. East Hanover, NJ: Novartis Inc; 2019.

6. Dugel PU, Koh A, Ogura Y, et al. HAWK and HARRIER: Phase 3, multicenter, randomized, double-masked trials of brolucizumab for neovascular age-related macular degeneration. Ophthalmology. 2020;127(1):72-84. doi:10.1016/j.ophtha.2019.04.017

7. Yannuzzi NA, Freund KB. Brolucizumab: evidence to date in the treatment of neovascular age-related macular degeneration. Clin Ophthalmol. 2019;13:1323-1329. doi:10.2147/OPTH.S184706

8. Elsing SH, Postel EA, Gill MK, Jampol LM, Jaffe GJ. Management of eyes with both idiopathic macular hole and choroidal neovascularization. Retina. 2001;21(6):613-618. doi:10.1097/00006982-200112000-00008

9. Gaudreault J, Gunde T, Floyd HS. Preclinical pharmacology and safety of ESBA1008, a single-chain antibody fragment, investigated as potential treatment for age related macular degeneration. Invest Ophthalmol Vis Sci. 2012;53(14):3025.

10. Semeraro F, Morescalchi F, Duse S, Parmeggiani F, Gambicorti E, Costagliola C. Aflibercept in wet AMD: specific role and optimal use. Drug Des Devel Ther. 2013;7:711-722. doi:10.2147/DDDT.S40215 
11. Goto E, Yagi Y, Matsumoto Y, Tsubota K. Impaired functional visual acuity of dry eye patients. Am J Ophthalmol. 2002;133(2):181-186. doi:10.1016/S0002-9394(01)01365-4

12. Kaiser PK. Prospective evaluation of visual acuity assessment: a comparison of snellen versus ETDRS charts in clinical practice (An AOS Thesis). Trans Am Ophthalmol Soc. 2009;107:311-324.

13. Shima C, Sakaguchi H, Gomi F, et al. Complications in patients after intravitreal injection of bevacizumab. Acta Ophthalmol (Copenh). 2008;86:372-376. doi:10.1111/j.1600-0420.2007.01067.x

14. Souied EH, Dugel PU, Ferreira A, Hashmonay R, Lu J, Kelly SP. Severe ocular inflammation following ranibizumab or aflibercept injections for age-related macular degeneration: a retrospective claims database analysis. Ophthalmic Epidemiol. 2016;23(2):71-79. doi:10.3109/09286586.2015.1090004
15. Rajan K. Update: brolucizumab's safety under review. Am Acad Ophthalmol. 2020. Available from: https://www.aao.org/headline/bro lucizumab-s-safety-under-review. Accessed March 1, 2020.

16. Yu JH, Gallemore E, Kim JK, Patel R, Calderon J, Gallemore RP. Silicone oil droplets following intravitreal bevacizumab injections. Am J Ophthalmol Case Rep. 2017;10:142-144. doi:10.1016/j. ajoc.2017.07.009

\section{Publish your work in this journal}

The International Medical Case Reports Journal is an international, peer-reviewed open-access journal publishing original case reports from all medical specialties. Previously unpublished medical posters are also accepted relating to any area of clinical or preclinical science. Submissions should not normally exceed 2,000 words or 4 published pages including figures, diagrams and references. The manuscript management system is completely online and includes a very quick and fair peer-review system, which is all easy to use. Visit http://www.dovepress.com/testimonials.php to read real quotes from published authors. 